\title{
Evasion of adaptive immunity by HIV through the action of host APOBEC3G/F enzymes
}

\author{
Michael Grant ${ }^{*}$ and Mani Larijani*
}

\begin{abstract}
APOBEC3G (A3G) and APOBEC3F (A3F) are DNA-mutating enzymes expressed in T cells, dendritic cells and macrophages. A3G/F have been considered innate immune host factors, based on reports that they lethally mutate the HIV genome in vitro. In vivo, A3G/F effectiveness is limited by viral proteins, entrapment in inactive complexes and filtration of mutations during viral life cycle. We hypothesized that the impact of sub-lethal A3G/F action could extend beyond the realm of innate immunity confined to the cytoplasm of infected cells. We measured recognition of wild type and A3G/F-mutated epitopes by cytotoxic T lymphocytes (CTL) from HIV-infected individuals and found that A3G/F-induced mutations overwhelmingly diminished CTL recognition of HIV peptides, in a human histocompatibility-linked leukocyte antigen (HLA)-dependent manner. Furthermore, we found corresponding enrichment of A3G/Ffavored motifs in CTL epitope-encoding sequences within the HIV genome. These findings illustrate that A3G/F-mediated mutations mediate immune evasion by HIV in vivo. Therefore, we suggest that vaccine strategies target $T$ cell or antibody epitopes that are not poised for mutation into escape variants by A3G/F action.
\end{abstract}

\section{Background}

The classical view of $\mathrm{A} 3 \mathrm{G} / \mathrm{F}$ as anti-viral host restriction factors and innate immune agents

APOBEC3G (A3G) and APOBEC3F (A3F) are members of the apolipoprotein $B$ mRNA-editing enzyme catalytic polypeptide-like editing complex (APOBEC) family of cytidine deaminase enzymes. This family includes activation-induced cytidine deaminase (AID), APOBEC1, APOBEC2, APOBEC3A-H, and APOBEC4. All APOBEC3 (A3) sub-branch members (A3A, A3B, A3F, A3G and A3H) exhibit varying degrees of anti-viral restriction activity, though A3G and A3F are the most potent restrictors of HIV. For this reason, the mechanisms of A3G/F have been the subject of intense study over the last decade. From these studies, it is appreciated that $\mathrm{A} 3 \mathrm{G} / \mathrm{F}$ mutate cytidine $(\mathrm{C})$ to uridine $(\mathrm{U})$ in trinucleotide mutational hotspots $(\mathrm{CCC}-$ which is GGG on the coding strand sequence-and less often TCC for

\footnotetext{
*Correspondence: mgrant@mun.ca; mlarijani@mun.ca

Program in Immunology and Infectious Diseases, Division of Biomedical Sciences, Faculty of Medicine, Memorial University of Newfoundland, St. John's, NF A1B 3V6, Canada
}

A3G; TTC for A3F) in the reverse transcribed DNA copy of HIV, registering as guanine $(\mathrm{G})$ to adenine (A) substitutions in the plus strand DNA [1-6]. These mutations can trigger DNA degradation or lead to stop codons, frame shift or mutated viral proteins. In addition to hindering HIV through their mutational activities, A3G/F can also physically sequester the viral RNA, block reverse transcriptase (RT) and obstruct integration into the host cell genome [7-9].

Since their discovery early in the last decade, the prevalent view of A3G/F has been as anti-retroviral restriction factors. This is because, first A3G/F significantly diminishes viral propagation in several in vitro or ex vivo reporter systems of HIV infection [1, 3, 6, 10]. Second, in said reporter systems of HIV infection and in biochemical assays, A3G/F introduce high levels of $\mathrm{G}$ to $\mathrm{A}$ mutations in viral genomes, in the range of hundred mutations/ genome/replication round, orders of magnitude higher than HIV's own reverse transcriptase (RT), which introduces approximately one mutation in the $10 \mathrm{~kb}$-long HIV genome per replication cycle [11]. Third, the expansion of a single APOBEC3 gene in mice to 7 in primates and 
the high divergence within A3 branch amongst primates have been suggested to be concomitant with the emergence of modern lentiviruses, and interpreted as evidence for the important role that this family of enzymes plays in anti-retroviral immunity [12].

A3G/F have been considered as innate immune agents, because they are constitutively expressed in CD4+ T lymphocytes, macrophages, and dendritic cells regardless of infection status, though inflammatory cytokines can upregulate their expression post-infection [13]. Second, they non-discriminately mutate any cytoplasmic DNA, without sequence or antigenic specificity. It is well established that in the long-term, it is adaptive immunity that controls HIV infection. The antibody and CTL responses are key: presence of broadly neutralizing antibodies and the robustness of anti-HIV CTL are the two cornerstones of a successful anti-HIV immune response, and correlate inversely with disease progression [14-21]. Thus, in the mainstream view of A3G/F as innate immunity's antiviral host restriction factors, A3G/F have been proposed to serve as a first line of defense to limit viral replication, whilst the HIV-specific adaptive immune responses of CTL and antibodies take weeks to months to ramp up to effective levels.

\section{A novel role for $A 3 G / F$ beyond innate immunity and in adaptive immunity}

In contrast to lethal mutation of HIV's genome and its effective restriction by $A 3 G / F$ in various in vitro cell culture-based assays of HIV infectivity, A3G/F activity in vivo only leads to low level non-lethal mutations of the viral genome. There are several reasons for this: first, the Virion infectivity factor (Vif) protein of HIV binds and targets A3G/F for degradation via a ubiquitin-dependent proteosomal pathway. Thus, when Vif is present, A3G/F effectiveness in viral restriction is severely diminished, and experimental systems where A3G/F can functionally restrict HIV use Vif-deficient viruses F [22-27]. Second, due to their highly charged surfaces and propensity for non-specific DNA/RNA or protein-protein binding, most cellular A3G/F are rendered inactive due to entrapment in mega-dalton sized ribonuclear cytoplasmic highmolecular-mass complexes (HMM) [28]. Third, even in cells where A3G/F introduce a high mutational load on the invading viral genomes, their final mutational signature in synthesized virions is much reduced due to the inherent selection that successive stages of viral replication impose for fit viral genomes [29].

Since most HIV genomes would only suffer low level A3G/F mutations levels, we and others proposed that non-lethal levels of A3G/F-induced mutations could impact adaptive immunity in HIV-infected individuals through modification of specific epitopes [30]. Two earlier studies had examined the role of APOBECinduced mutations on recognition of HIV CTL epitopes, using a bioinformatics approach and a mouse model, respectively [31, 32]. The former study reported that approximately one-third of rapidly diversifying regions of HIV mediating CTL escape are embedded in A3G hotspots and suggested that A3G/F action may aid HIV by mutating its CTL epitopes towards immune evasion. In contrast, the latter study reported that A3G mutations enhanced the virus-specific CTL response through the introduction of premature stop codons in the HIV genome that cause the generation of truncated or misfolded proteins. In this study, Vif + or Vif- HIV was produced in the presence or absence of A3G in a cell line and subsequently used to infect peripheral blood mononuclear cells (PBMCs) followed by assessing their susceptibility to being killed by CTL bearing transgenic peptide-specific $\mathrm{T}$ cell receptors. This was the first study to show that in principle, the impact of $\mathrm{A} 3 \mathrm{G} / \mathrm{F}$ mutations could extend to the adaptive immune realm. Nevertheless, whether this might be the case in HIV-infected humans had not been examined.

We sought to determine if $\mathrm{A} 3 \mathrm{G} / \mathrm{F}$ mutations play a role in adaptive immunity in HIV-infection. To this end, we measured CTL recognition of wild type and A3G/Fmutated epitopes in a cohort of HIV-infected individuals. First, we cataloged all CTL epitopes in HIV proteins using the experimentally-verified HIV molecular immunology database, and identified CTL epitopes whose encoding sequences contain A3G/F-mutable hotspots. Next, we synthesized wild type and A3G/F-mutated peptide CTL epitopes restricted to various Class I HLA molecules and evaluated CTL recognition of wild type and A3G/F-mutated CTL epitopes by CTL from HLAmatched subjects. We found that in contrast to control peptides mutated in sequences outside A3G/F hotspots, A3G/F-induced mutations overwhelmingly diminished CTL recognition of HIV peptides [33].

Two further key observations can be taken as evidence for the physiological significance of A3G/F-mediated CTL evasion by HIV. First, we found that HIV's genome has enriched for A3G/F mutational hotspots in sequences encoding CTL epitopes, either located within more immunogenic polypeptides (Gag, Pol and Nef), or restricted to class I HLA molecules that present highly immunogenic epitopes (e.g. HLA-B57) [33]. Second, we observed strong HLA specificity: for epitopes restricted to HLA-B57 and A2, A3G/F mutations significantly decreased CTL recognition [34]. In contrast, A3G/F mutations enhanced the CTL recognition of most HLA-B35-restricted epitopes, and a very limited subset of epitopes restricted to HLA-A2, A3, B44 and B57. This HLA specificity, namely the prevalence of escape 
mutations linked to HLA alleles that present immunogenic CTL epitopes, such as HLA-B57, fits closely with the pattern of well-characterized CTL escape variants in the population $[35,36]$. Thus, the evolution of the viral genome to position more A3G/F target hotspots in immunogenic CTL epitopes, and the HLA specificity strongly suggest that A3G/F-mediated CTL escape is a significant aspect of HIV infection in vivo.

\section{Conclusions and implications for vaccine design}

It is likely that A3G/F do indeed impact adaptive immunity in HIV-infected individuals, and that A3G/F-mediated CTL escape is a significant phenomenon in HIV infection. We note that a parallel role for A3G/F in mediating drug resistance mutations of the viral genome has already been demonstrated. Together, these results indicate that A3G/F carry significant pro-retroviral activities.

It will be important to understand the extent of interindividual variation in A3G/F-mediated CTL escape during HIV infection, to gain mechanistic insights into the molecular and cellular pathways through which A3G/Fmediated mutations drive CTL escape, and compare these to known pathways of immune evasion for HIV and other viruses.

In the future, it will also be important to fully define the rare set of CTL epitopes that we found are either not transformed into escape variants, or conversely, are made more immunogenic by A3G/F-mediated mutations. Our work suggests that any vaccination strategy, whether eliciting CTL or antibody responses, can be rendered more effective if these "A3G/F-resistant" or "A3G-enhanced" epitopes are taken into consideration.

\section{Authors' contributions}

ML and MG wrote and edited this manuscript. Both authors read and approved the final manuscript.

\section{Competing interests}

The authors declare that they have no competing interests.

\section{Availability of data and materials}

Not applicable.

\section{Consent for publication}

Not applicable.

\section{Ethics approval and consent to participate}

Not applicable.

\section{Funding}

The work reviewed here was supported by CIHR (MOP111132 and OCH131580) and Canadian Foundation for AIDS Research (CANFAR) operating grants to ML, and by CIHR operating Grants (HOP-111725, HOP-93428) and a ClHR catalyst Grant (HC1-112568) to MG.

\section{Publisher's Note}

Springer Nature remains neutral with regard to jurisdictional claims in published maps and institutional affiliations.
Received: 15 March 2017 Accepted: 11 August 2017

Published online: 12 September 2017

\section{References}

1. Harris RS, Bishop KN, Sheehy AM, Craig HM, Petersen-Mahrt SK, Watt IN, Neuberger MS, Malim MH. DNA deamination mediates innate immunity to retroviral infection. Cell. 2003:113(6):803-9.

2. Klarmann GJ, Chen X, North TW, Preston BD. Incorporation of uracil into minus strand DNA affects the specificity of plus strand synthesis initiation during lentiviral reverse transcription. J Biol Chem. 2003;278(10):7902-9.

3. Lecossier D, Bouchonnet F, Clavel F, Hance AJ. Hypermutation of HIV-1 DNA in the absence of the Vif protein. Science. 2003;300(5622):1112.

4. Mangeat B, Turelli P, Caron G, Friedli M, Perrin L, Trono D. Broad antiretroviral defence by human APOBEC3G through lethal editing of nascent reverse transcripts. Nature. 2003;424(6944):99-103.

5. Zhang H, Yang B, Pomerantz RJ, Zhang C, Arunachalam SC, Gao L. The cytidine deaminase CEM15 induces hypermutation in newly synthesized HIV-1 DNA. Nature. 2003:424(6944):94-8.

6. Yu Q, Konig R, Pillai S, Chiles K, Kearney M, Palmer S, Richman D, Coffin $J M$, Landau NR. Single-strand specificity of APOBEC3G accounts for minus-strand deamination of the HIV genome. Nat Struct Mol Biol. 2004;11(5):435-42.

7. Belanger K, Savoie M, Rosales Gerpe MC, Couture JF, Langlois MA. Binding of RNA by APOBEC3G controls deamination-independent restriction of retroviruses. Nucleic Acids Res. 2013;41(15):7438-52.

8. Guo F, Cen S, Niu M, Yang Y, Gorelick RJ, Kleiman L. The interaction of APOBEC3G with human immunodeficiency virus type 1 nucleocapsid inhibits tRNA3Lys annealing to viral RNA. J Virol. 2007;81 (20):11322-31.

9. Adolph MB, Webb J, Chelico L. Retroviral restriction factor APOBEC3G delays the initiation of DNA synthesis by HIV-1 reverse transcriptase. PLoS ONE. 2013;8(5):e64196.

10. Harris RS. Enhancing immunity to HIV through APOBEC. Nat Biotechnol. 2008;26(10):1089-90.

11. Preston BD, Poiesz BJ, Loeb LA. Fidelity of HIV-1 reverse transcriptase. Science. 1988;242(4882):1168-71.

12. Zhang J, Webb DM. Rapid evolution of primate antiviral enzyme APOBEC3G. Hum Mol Genet. 2004:13(16):1785-91.

13. Koning FA, Newman EN, Kim EY, Kunstman KJ, Wolinsky SM, Malim MH. Defining APOBEC3 expression patterns in human tissues and hematopoietic cell subsets. J Virol. 2009:83(18):9474-85.

14. Wibmer CK, Moore PL, Morris L. HIV broadly neutralizing antibody targets. Curr Opin HIV AIDS. 2015;10(3):135-43.

15. Borrow P, Lewicki H, Hahn BH, Shaw GM, Oldstone MB. Virus-specific CD8 + cytotoxic T-lymphocyte activity associated with control of viremia in primary human immunodeficiency virus type 1 infection. JVirol. 1994;68(9):6103-10

16. Heintel T, Sester M, Rodriguez MM, Krieg C, Sester U, Wagner R, Pees HW, Gartner B, Maier R, Meyerhans A. The fraction of perforin-expressing HIVspecific CD8 T cells is a marker for disease progression in HIV infection. Aids. 2002;16(11):1497-501.

17. Shankar P, Russo M, Harnisch B, Patterson M, Skolnik P, Lieberman J. Impaired function of circulating HIV-specific CD8(+) T cells in chronic human immunodeficiency virus infection. Blood. 2000;96(9):3094-101.

18. Ogg GS, Jin X, Bonhoeffer S, Dunbar PR, Nowak MA, Monard S, Segal JP, Cao Y, Rowland-Jones SL, Cerundolo V, et al. Quantitation of HIV1-specific cytotoxic T lymphocytes and plasma load of viral RNA. Science. 1998:279(5359):2103-6.

19. Rinaldo C, Huang XL, Fan ZF, Ding M, Beltz L, Logar A, Panicali D, Mazzara G, Liebmann J, Cottrill M, et al. High levels of anti-human immunodeficiency virus type 1 (HIV-1) memory cytotoxic T-lymphocyte activity and low viral load are associated with lack of disease in HIV-1-infected longterm nonprogressors. J Virol. 1995;69(9):5838-42.

20. Harrer T, Harrer E, Kalams SA, ElbeikT, Staprans SI, Feinberg MB, Cao Y, Ho DD, Yilma T, Caliendo AM, et al. Strong cytotoxic T cell and weak neutralizing antibody responses in a subset of persons with stable nonprogressing HIV type 1 infection. AIDS Res Hum Retroviruses. 1996;12(7):585-92.

21. Emu B, Sinclair E, Hatano H, Ferre A, Shacklett B, Martin JN, McCune JM, Deeks SG. HLA class I-restricted T-cell responses may contribute 
to the control of human immunodeficiency virus infection, but such responses are not always necessary for long-term virus control. J Virol. 2008;82(11):5398-407.

22. Kao S, Akari H, Khan MA, Dettenhofer M, Yu XF, Strebel K. Human immunodeficiency virus type 1 Vif is efficiently packaged into virions during productive but not chronic infection. J Virol. 2003;77(2):1131-40.

23. Yu Y, Xiao Z, Ehrlich ES, Yu X, Yu XF. Selective assembly of HIV-1 Vif-Cul5ElonginB-ElonginC E3 ubiquitin ligase complex through a novel SOCS box and upstream cysteines. Genes Dev. 2004;18(23):2867-72.

24. Santa-Marta M, da Silva FA, Fonseca AM, Goncalves J. HIV-1 Vif can directly inhibit apolipoprotein B mRNA-editing enzyme catalytic polypeptide-like 3G-mediated cytidine deamination by using a single amino acid interaction and without protein degradation. J Biol Chem. 2005;280(10):8765-75.

25. Goncalves J, Santa-Marta M. HIV-1 Vif and APOBEC3G: multiple roads to one goal. Retrovirology. 2004;1:28.

26. Santa-Marta M, Aires da Silva F, Fonseca AM, Rato S, Goncalves J. HIV-1 Vif protein blocks the cytidine deaminase activity of B-cell specific AID in $E$. coli by a similar mechanism of action. Mol Immunol. 2007;44(4):583-90.

27. Yu X, Yu Y, Liu B, Luo K, Kong W, Mao P, Yu XF. Induction of APOBEC3G ubiquitination and degradation by an HIV-1 Vif-Cul5-SCF complex. Science. 2003:302(5647):1056-60.

28. Chiu YL. Biochemical fractionation and purification of high-molecularmass APOBEC3G complexes. Methods Mol Biol. 2011;718:185-206.

29. Russell RA, Moore MD, Hu WS, Pathak VK. APOBEC3G induces a hypermutation gradient: purifying selection at multiple steps during HIV-1 replication results in levels of G-to-A mutations that are high in DNA, intermediate in cellular viral RNA, and low in virion RNA. Retrovirology. 2009;6:16.
30. Monajemi M, Woodworth CF, Benkaroun J, Grant M, Larijani M. Emerging complexities of APOBEC $3 G$ action on immunity and viral fitness during HIV infection and treatment. Retrovirology. 2012;9:35.

31. Wood N, Bhattacharya T, Keele BF, Giorgi E, Liu M, Gaschen B, Daniels M, Ferrari G, Haynes BF, McMichael A, et al. HIV evolution in early infection: selection pressures, patterns of insertion and deletion, and the impact of APOBEC. PLoS Pathog. 2009;5(5):e1000414.

32. Casartelli N, Guivel-Benhassine F, Bouziat R, Brandler S, Schwartz O, Moris A. The antiviral factor APOBEC3G improves $C T L$ recognition of cultured HIV-infected T cells. J Exp Med. 2010;207(1):39-49.

33. Monajemi M, Woodworth CF, Zipperlen K, Gallant M, Grant MD, Larijani M. Positioning of APOBEC3G/F mutational hotspots in the human immunodeficiency virus genome favors reduced recognition by $C D 8+T$ cells. PLoS ONE. 2014;9(4):e93428.

34. Squires KD, Monajemi M, Woodworth CF, Grant MD, Larijani M. Impact of APOBEC mutations on CD8 + T cell recognition of HIV epitopes varies depending on the restricting HLA. J Acquir Immune Defic Syndr. 2015:70(2):172-8

35. Allen TM, Altfeld M, Geer SC, Kalife ET, Moore C, O'Sullivan KM, Desouza I, Feeney ME, Eldridge RL, Maier EL, et al. Selective escape from $\mathrm{CD} 8+\mathrm{T}$-cell responses represents a major driving force of human immunodeficiency virus type 1 (HIV-1) sequence diversity and reveals constraints on HIV-1 evolution. JVirol. 2005;79(21):13239-49.

36. Altfeld M, Kalife ET, Qi Y, Streeck H, Lichterfeld M, Johnston MN, Burgett N, Swartz ME, Yang A, Alter G, et al. HLA alleles associated with delayed progression to AIDS contribute strongly to the initial CD8(+) T cell response against HIV-1. PLoS Med. 2006;3(10):e403.

\section{Submit your next manuscript to BioMed Central and we will help you at every step:}

- We accept pre-submission inquiries

- Our selector tool helps you to find the most relevant journal

- We provide round the clock customer support

- Convenient online submission

- Thorough peer review

- Inclusion in PubMed and all major indexing services

- Maximum visibility for your research

Submit your manuscript at www.biomedcentral.com/submit
() Biomed Central 